Carnets de géographes

GÉOGRAPHES.

$7 \mid 2014$

Les espaces de l'entre-deux

\title{
Sans parler du chien ou comment nous retrouvâmes enfin la potiche de l'évêque
}

To say nothing of the dog, bantam spectra books, new York, 1997

\section{Morwenna Coquelin}

\section{(2) OpenEdition}

\section{Journals}

Édition électronique

URL : http://journals.openedition.org/cdg/502

DOI : $10.4000 /$ cdg. 502

ISSN : 2107-7266

\section{Éditeur}

UMR 245 - CESSMA

\section{Référence électronique}

Morwenna Coquelin, «Sans parler du chien ou comment nous retrouvâmes enfin la potiche de

l'évêque ", Carnets de géographes [En ligne], 7 | 2014, mis en ligne le 01 décembre 2014, consulté le 24 septembre 2020. URL : http://journals.openedition.org/cdg/502 ; DOI : https://doi.org/10.4000/cdg. 502

\section{c) (i) $९$}

La revue Carnets de géographes est mise à disposition selon les termes de la Licence Creative Commons Attribution - Pas d'Utilisation Commerciale - Pas de Modification 4.0 International. 


\title{
SANS PARLER DU CHIEN OU COMMENT NOUS RETROUVÂMES ENFIN LA POTICHE DE L'EVÊQUE, CONNIE WILLIS, J'AI LU, PARIS, 2003 (TO SAY NOTHING OF THE DOG, BANTAM SPECTRA BOOKS, NEW YORK, 1997).
}

\author{
MORWENNA COQUELIN \\ Agrégée d'histoire, doctorante \\ EHESS, Centre de Recherches Historiques, Gahom \\ mocoquelin@gmail.com
}

L'entre-deux semble indéniablement géographique, qu'il s'agisse d'un espace intermédiaire, d'un espace particulier lié au voyage ou à la connexion, d'une interface. C'est bien une position, que signale l'inconfortable expression du cul et des deux chaises. Une telle "position" semble impossible d'un point de vue temporel, puisqu'on ne peut guère connecter des temps qui n'existent justement pas en même temps, contrairement aux espaces parallèles, mais en une succession où ils s'excluent toujours les uns les autres.

Pourtant ces "entre-deux temporels " existent à condition que l'on veuille bien accepter l'une ou l'autre de ces conventions : soit la possibilité de voyager dans le temps, vers l'avant ou vers l'arrière, soit la concomitance d'univers parallèles dans lesquels le temps se déroule de façon décalée. C'est là toute la base d'une bonne partie de la science-fiction que de postuler le saut d'un temps à un autre et de devoir donc résoudre la question du " comment ": de façon technique - et ce sont les pages, parfois longues, décrivant les mécanismes physiques et les technologies permettant ces sauts -, de façon "physique ", avec la description des interfaces créées par les machines à voyager dans le temps, véritables portes sur un autre monde, de façon normative avec le problème récurrent des impossibilités, anachronismes, ruptures et accrocs qu'il convient de ne pas produire sous peine de changer tout le futur.

Dans Sans parler du chien, ou comment nous retrouvâmes la potiche de l'évêque Connie Willis fait du voyage dans le temps la base littérale du travail d'historiens de l'Université d'Oxford en 2057. Ces derniers ne sont plus contraints par les horaires d'ouverture des archives mais se rendent directement sur place en missions d'observation. Après le temps des héros dans les années 2000-2020, période où l'historien, aventurier ne sachant guère où il atterrirait ni s'il reviendrait, menait une recherche semblable à l'exploration du monde du

\footnotetext{
${ }^{1}$ Toutes les références sont faites ici à partir de la traduction de Jean-Pierre Pugi parue aux Editions J'ai Lu en 2003.
} 
temps où les cartes n'étaient pas complètes (p. 451), est venu le temps presque routinier, quoiqu'épuisant lorsqu'il faut obéir aux désirs d'une mécène perfectionniste et sans pitié, où l'historien saute dans le passé comme son homologue parisien et bien réel de 2014 se rend à la BnF. Pour parachever la reconstruction de la cathédral de Coventry telle qu'elle était avant le raid de 1940 qui la détruisit, Ned Henry parcourt le passé à la recherche du dernier objet manquant, la potiche de l'évêque. Passablement épuisé par trop de sauts en trop peu de temps, il est expédié dans l'Angleterre victorienne comme dans une zone hors d'atteinte des ennuis présents, et chargé par la même occasion de rattraper l'acte irréfléchi de sa collègue, la très séduisante Verity Kindle, qui a ramené contre toutes les lois temporelles un chat espèce éteinte depuis 2004. Elle-même avait été envoyée à Coventry dans les années 1880 pour tenter d'assister à un événement dont on a perdu les détails mais dont on sait qu'il est fondamental dans l'histoire de la potiche. La cure de repos de Ned se transforme donc en mission d'assistance auprès de Verity, bien que le déphasage dont souffre Ned rende son jugement assez flottant et occasionne diverses modifications du passé que les deux historiens s'évertuent à résoudre pour rétablir le continuum temporel.

Sans parler du chien mêle ainsi anticipation et roman historique ; c'est aussi l'occasion d'une réflexion sur le métier d'historien (p. 71) et les conditions de la recherche, puisqu'en 2057 comme aujourd'hui l'argent est le nerf de la guerre et le généreux mécène qui finance peut aussi devenir le tyrannique patron qui oriente les chantiers (p. 45). Plus généralement, le roman s'interroge sur le déroulement du temps et le rôle des acteurs dans la fabrique de l'histoire et l'inflexion des événements, par le biais d'une querelle entre deux professeurs victoriens dont l'un soutient la primauté du rôle des individus et l'autre en tient pour les "forces naturelles" (p. 108). Dès l'arrivée de Ned Henry dans un monde plein de quiproquos, le lecteur est ainsi mis en contact avec des questions épistémologiques, celle de la place des individus au sein d'une histoire collective et celle des causalités en histoire.

L'entre-deux le plus évident dans l'exploration temporelle est la zone de passage, située, dans le passé, en des lieux à l'abri des regards, et en 2057 dans les laboratoires de I'Université. La porte, qui s'ouvre par intermittence selon des intervalles prédéfinis en 2057 et dont l'ouverture prend la forme d'un miroitement, est l'interface par excellence, entredeux spatial autant que temporel puisque c'est le déplacement de et vers Oxford mais aussi le franchissement de quelques jours à quelques siècles le temps d'un baiser. Le premier devoir d'un historien est ainsi devenu de "s'assurer de ses coordonnées spatiotemporelles " afin de vérifier l'absence d'erreurs et, au besoin, de protéger sa vie. Semblable à son ancêtre ou à son double du monde réel, l'historien de 2057 continue à s'appuyer sur les coiffures pour déterminer l'époque dans laquelle il est arrivé, en cas de dérèglement et d'imprévu, avec la même incertitude souvent qui caractérise souvent la datation des statues antiques ; il a lui-même dû adopter une coupe de cheveux dont la longueur intermédiaire lui permet de passer inaperçu à peu près à toute époque.

L'entre-deux caractérise aussi la situation du visiteur qui n'est plus dans son époque sans pour autant appartenir, malgré la qualité de sa préparation, à celle dans laquelle il évolue momentanément. Ainsi nombre de lieux et de moments sont-ils interdits aux historiens noirs, qui y risqueraient par trop leur vie. Mais même si Verity Kindle a toute l'apparence de la demoiselle victorienne de bonne famille, elle n'en a pas toujours les réactions et le voyage dans le temps oblige à une discipline ferme pour brider la spontanéité. Sans compter les 
étourderies anachroniques, évocation d'un naufrage célèbre 24 ans trop tôt, citation d'un poème non encore écrit ou oubli dans un coin d'un vêtement non encore inventé.

En cela l'historien de 2057 ne diffère pas de son confrère de 2014 et du monde réel - c'est bien le propre de la science fiction que de transposer notre monde dans une apparente étrangeté qui sert avant tout à souligner les caractéristiques du présent. Les archives sont en effet un moment où l'on n'est ni là ni ailleurs, où le temps s'écoule selon le rythme particulier de la paléographie et des tâtonnements dans des salles ouvertes fort peu et de façon souvent fort malcommode. L'historien y parcourt le temps dans les deux sens, se plongeant dans l'avant et revenant vers le maintenant, mais toujours en esprit, et toujours vers un point d'arrivée déjà connu et donné, ce qui limite considérablement l'incertitude auquel le voyageur est soumis. Ce va-et-vient permanent provoque une temporalité propre à l'étude et au chercheur et permet aussi d'objectiver le temps. Et tout comme l'historienvoyageur de 2057, décalé parfois dans son nouvel environnement et souvent déphasé à force de sauts trop nombreux vers le passé et d'une extension par trop grande de son temps ${ }^{2}$, son compère en archives peut vivre des moments de flottement induits par l'étrangeté de l'écriture, de l'orthographe, voire de la langue, et des idées exprimées. Lui aussi est dans un entre-deux, entre maintenant et alors, un entre-deux forgé par la concentration, l'immersion dans les documents mais aussi une immobilité physique à peine rompue par les pauses éventuelles ou le tortillement sur un mobilier pas toujours éminemment confortable.

La perception du temps est accrue dans un monde où il peut se parcourir. II peut se répéter, pour rattraper des erreurs ou profiter d'une avancée technologique nouvelle permettant enfin de résoudre certaines énigmes - le voyage dans le temps permet de rompre avec l'éphémère et l'inéluctabilité du temps qui passe, et est porteur d'espoir puisque tout est recommençable et perfectible (p. 433). Le voyage dans le passé peut aussi permettre de gagner du temps alors même que, dans l'instant présent, on attend de toujours pouvoir repartir assez en arrière pour être dans les temps. Mais cela ne vaut que pour ce qui affecte le groupe: I'individu lui-même est toujours soumis à l'insoutenable fluidité du temps qui s'écoule, et ne peut jamais revivre ce qu'il a vécu, que ce soit dans son présent ou dans le passé. Plusieurs temps coexistent ainsi : un temps externe au voyageur et son propre temps, un temps linéaire qui doit se dérouler comme il s'est déjà déroulé et un temps non pas cyclique, puisque l'homme ne revit pas les événements, mais hélicoïdal, constitué des boucles répétées formées par les sauts. Le chercheur est alors en proie à la mélancolie qui poursuit l'homme, mortel destiné à toujours voir s'effacer les moments qu'il vit sitôt qu'il les a vécus, ou voyageur sans cesse entre deux époques et confronté en permanence à l'inaccessibilité du passé par la rencontre avec des êtres en réalité toujours déjà morts pour lui : « elle incarnait la jeunesse et la beauté alors que je la savais depuis longtemps mariée à Terrence, morte et enterrée dans un cimetière semblable à celui que j'apercevais au sommet de la colline " ( $p$ 117-118). Le voyage dans le passé ne peut être une véritable rencontre, puisque les hommes du passé ne savent jamais à qui ils ont réellement affaire, et n'est pas non plus un moment en propre puisque les historiens n'appartiennent pas à l'époque où ils vivent provisoirement, ne sont que spectateurs et n'agissent qu'en vue

\footnotetext{
${ }^{2}$ Les trop nombreux sauts provoquent de plus un déphasage qui plonge l'historien dans un inconfortable état de fatigue extrême, de pensée lente, de faiblesse des sens mais d'acuité de la sensibilité qui se manifeste par un lyrisme quelque peu larmoyant.
} 
d'événements déjà définis et réglés. L'entre-deux est l'espace de la nostalgie, douloureux désir, et, quand bien même l'homme semble s'être échappé de sa condition de prisonnier du temps, il y est violemment rappelé par les règles du voyage qui interdisent le transport des êtres et des objets venus du passé. Bien que de chair et de sang, les êtres rencontrés par I'historien-voyageur sont aussi mités que les archives et tout aussi près de tomber en poussière que des vestiges archéologiques trop vite sortis du sol. La recherche en archives aussi autorise à toucher du doigt, littéralement, le passé, sans pour autant y participer. C'est $\mathrm{n}^{\prime}$ avoir entre les mains que la trace, le fossile.

L'interface des chercheurs de Sans parler du chien n'est d'ailleurs pas totalement poreuse et manifeste paradoxalement l'incommunicabilité fondamentale entre les temps: outre la barrière culturelle que les années d'études - et parfois des bandes subliminales - aident à franchir, il existe une barrière physique, même si elle n'opère que dans un sens. II est impossible de rapporter quoi que ce soit du passé révolu, qui n'est pas transportable: l'interface détecte tout objet et refuse le passage, de même que l'archiviste veille à ce que nul ne parte avec un chartrier sous le bras ou ne prenne à la dérobée des photographies encore souvent interdites. Les raisons invoquées dans le roman sont sanitaires mais il s'agit aussi de ne pas rompre le fragile déroulement du temps. Quand l'un des héros réussit finalement à transporter un spécimen d'une espèce disparue, cela comble les besoins de ces savants de redonner à leur époque un peu de douceur et de charme, mais ce n'est qu'une saisie partielle du passé : la seule chose qui peut voyager vers le futur est sans importance ou sur le point de disparaître.

On risquerait autrement de modifier a posteriori l'histoire et le temps déjà écrit, de provoquer des "altérations ", des "incongruités ", des "panachronismes » (p. 44) ou de dangereuses évolutions du continuum (p. 245). Tout le roman est l'histoire d'une correction, à retardement, de la rupture provoquée par une historienne qui a dérogé à la loi élémentaire de tout voyage temporel: ne pas toucher. Mais les rebondissements de l'intrigue se révèlent au final moins les dérèglements d'un système altéré par des historiens trop peu scrupuleux que les "redondances " et " boucles de rétroaction " d'un "système chaotique » (p. 158) capable de se protéger lui-même et d'éliminer les interférences en modifiant les événements, même (surtout) mineurs pour assurer la pérennité des points sensibles, "pivots " de l'Histoire humaine (p. 60, p. 146). Ces pivots sont, eux, strictement inatteignables : les sauts des historiens vers ces destinations sont marqués par des décalages constants et irréductibles dans l'espace et le temps. Les portes s'y ferment de façon à éviter toute intervention future qui risquerait de réécrire l'histoire. Contrairement aux théories des historiens de 2057 et malgré toutes les simulations à partir de la bataille de Waterloo ${ }^{3}$, le " continuum " n'est pas un système linéaire (p. 44) - mais c'est cependant un système toujours déjà écrit quand le voyageur vers le passé s'y déplace. Son nom le marque bien, qui signale l'impossibilité des discontinuités et des interruptions. Le chemin diffèrera peut-être, mais la situation toujours déjà présente pour les historiens du futur sera atteinte. Est-ce le triomphe de la théorie du Professeur Overforce qui voit dans les forces de la nature, et non dans les acteurs, ce qui fait l'histoire ? Ou n'est-ce pas plutôt l'histoire qui devient un acteur capable de sentir les contraintes que l'on lui fait subir, et de les corriger par les mesures appropriées dont les hommes, y compris les historiens-voyageurs, ne sont que les

\footnotetext{
${ }^{3}$ Les historiens de 2057 ne font ici pas autre chose que l'historien de 2014 qui se demande ce qui aurait pu être, pour tenter de comprendre les mécanismes de ce qui s'est réellement passé.
} 
instruments? Le roman ne soumet pas pour autant la société humaine à un grand dessein qui la dépasserait. Comme dans tout voyage dans le temps, la seule contrainte est que se déroule toujours et encore ce qui s'est déjà déroulé : le temps peut être parcouru mais son écoulement reste irrévocable et l'on ne peut réécrire l'histoire: si des modifications marginales semblent possibles, elles n'ont en réalité aucune conséquence pour l'humanité dans son ensemble. Le fait que certains points soient hors d'atteinte pour les voyageurs du futur rend sans objet la tentation, ou le risque, de modifier les événements. Ce sont ces éléments que l'historien, réel cette fois, s'attache à repérer pour comprendre la logique des événements.

La lecture d'un roman où l'on voyage dans le temps oblige donc à se poser la question de ce qu'est le temps et du rapport que l'historien entretient avec lui, et c'est d'autant plus explicite dans Sans parler du chien que les héros sont des historiens devenus de véritables techniciens du temps. Le roman met en évidence la superposition des temps réels et virtuels : le présent des historiens, futur ignoré des acteurs qui sont étudiés ; le présent des acteurs, passé de l'historien, mais aussi son présent le temps d'un voyage plus ou moins bref ; le futur que les acteurs se rêvent ou craignent ; les fantasmes projetés sur le passé ; le présent télescopé d'un homme à la fois de 2057 et en 1888. L'historien doit cependant sortir de cet entre-deux pour se poser la question de sa place et respecter chacun des temps : si l'histoire s'écrit sans futur, l'historien est toujours en avance sur le temps dont il parle mais doit se garder de cette connaissance pour interpréter le passé téléologiquement. Son texte est le résultat du passage d'un temps à l'autre et de la mise en lumière de leur superposition mais l'auteur ne doit pas perdre sa place dans la boucle ou briser la disposition des moments. Ce qui n'empêche pas que Ned et Verity utilisent leur connaissance du futur et la passion victorienne pour le spiritisme afin de guider les acteurs vers ce qui leur apparait nécessaire à la réussite de leur mission et surtout à la protection du continuum qu'ils craignent d'avoir modifié.

Le roman d'anticipation montre que le temps doit être construit et objectivé, historicisé ; chaque couche appartient tour à tour au passé, au présent et au futur, et même les trois temps coexistent-ils puisque dans chaque moment les deux autres sont présents virtuellement à l'état de rêve, d'espoir, de souvenir ou d'attente. La réponse de Connie Willis évoque brillamment des questions épistémologiques et historiographiques au fil d'une intrigue à la construction impeccable et à l'écriture extrêmement drôle puisque la situation d'entre-deux est féconde sur le plan heuristique mais constitue parallèlement le préalable à tout humour grâce au décalage qu'elle induit. 